\title{
Water Absorption and FTIR Analysis of Three Type Natural Fiber Reinforced Composites
}

\author{
Xianyi Shao ${ }^{1, \text { a }}$, Luju He ${ }^{2, \text { b, }}$, Li Ma $^{3, \mathrm{c}}$ \\ ${ }^{1}$ College of Physics \& Electronic Engineering, Taizhou University, Taizhou 318000, China \\ ${ }^{2}$ Education and Information Technology Center, Guangdong University of Petrochemical \\ Technology, Maoming 525000, Guangdong, China \\ ${ }^{3}$ College of Mechanical and Electrical Engineering, Guangdong University of Petrochemical \\ Technology, Maoming 525000, Guangdong, China \\ asxy8718@163.com, ${ }^{\mathrm{b}}$ heluju@gdupt.edu.cn, ${ }^{\mathrm{c}}$ mali@gdupt.edu.cn
}

Keywords: Natural fibers; Water absorption; FTIR; Surface modification; Composite

Abstract. Surface treatment of three natural fibers including cellulose (CE), sawdust (SD) and wheat straw (WS) were carried out with triethoxysilane (AS), methacriloxy propyl trimethoxy silane (MS), and maleic anhydride grafted polypropylene (MAPP), respectively. Then the composites reinforced by these natural fibers were studied by water absorption and FTIR. It was found that water absorption decreased with employment of coupling agents. Among the coupling agents, MAPP exhibited the best performance in decreasing water absorption. In addition, FTIR analysis revealed the decrease in hydrophilicity of fibers with silane treatment and new bond formations with employment of MAPP.

\section{Introduction}

The use of natural fibers as a viable reinforcement in composite materials is steadily increasing and had gained a significant research interest over past few years, possibly due to biodegradability, low cost and abundance ${ }^{[1-2]}$. A major issue in achieving true reinforcement with the incorporation of lignocellulosic fibers into thermoplastic matrices is the inherent incompatibility between the hydrophilic fibers and the hydrophobic polymers ${ }^{[3-4]}$. This results in poor adhesion between fiber and matrix, and therefore in poor ability to transfer stress from the matrix to the fiber. To increase the quality of the fiber-matrix interface in composite materials, surface modification of fibers is required to achieve maximum compatibility and thereby good adhesion ${ }^{[5]}$. In this work, the effect of interfacial modification including triethoxysilane (AS) and methacriloxy propyl trimethoxy silane (MS), and maleic anhydride grafted polypropylene (MAPP) on water absorption of cellulose (CE), sawdust (SD) and wheat straw (WS) was investigated. In addition, FTIR analysis was used in order to characterize the chemical composition of fibers, PP and coupling agents. New bonds formed in the composites with the employment of coupling agents were also studied.

\section{Experimental}

Cellulose (CE), sawdust (SD) and wheat straw (WS) with fiber content of 10, 20, 30 and $40 \mathrm{wt} \%$ were treated with triethoxysilane (AS), methacriloxy propyl trimethoxy silane (MS), and maleic anhydride grafted polypropylene (MAPP), and then Chopped dried short untreated or treated fibers and PP pellets were compounded by using twin screw extruder for good mixing of fiber and polymer.

Water absorption of PP composites as a function of immersion time was determined following ASTM D570. Test specimen $\left(3 \times 1.75 \times 0.1 \mathrm{~cm}^{3}\right)$ was immersed in distilled water at room temperature. The percentage increase in weight during the immersion was calculated by the following equation:

$$
\text { Water absorption }(\%)=\left[\left(w-w_{0}\right) / w_{0}\right] \times 100 \%
$$

Where $w$ is wet weight and $w_{0}$ is dried weight. 
Fourier Transform Infrared (FTIR) study using an Attenuated Total Reflectance mode in the range of $400-4400 \mathrm{~cm}^{-1}$ was carried out using a Varian 800 -FTIR model scimitar series to study the function group.

\section{Results and discussion}

Water absorption. Fig. 1 shows effect of CE loading on water sorption of PP/CE composites. It is clearly seen that water sorption increases with increasing CE loading due to increasing hydrophilicity of the composite as expected. The increase is almost in a linear fashion. Linearity in the graph proves that the composites did not exhibit agglomeration which would complicate water sorption tendency of composites with increasing fiber loading. Fig. 2 illustrates the effect of coupling agent and fiber type on water sorption of composites at $30 \%$ fiber loaded composites. It was observed that three coupling agents decreased water sorption of composites at different levels for the three fiber types.

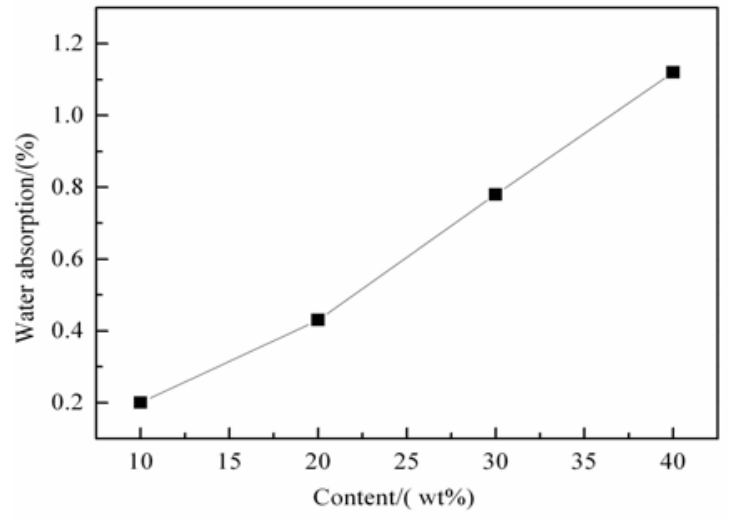

Fig. 1. Effect of CE loading on water sorption of $\mathrm{PP} / \mathrm{CE}$ composites

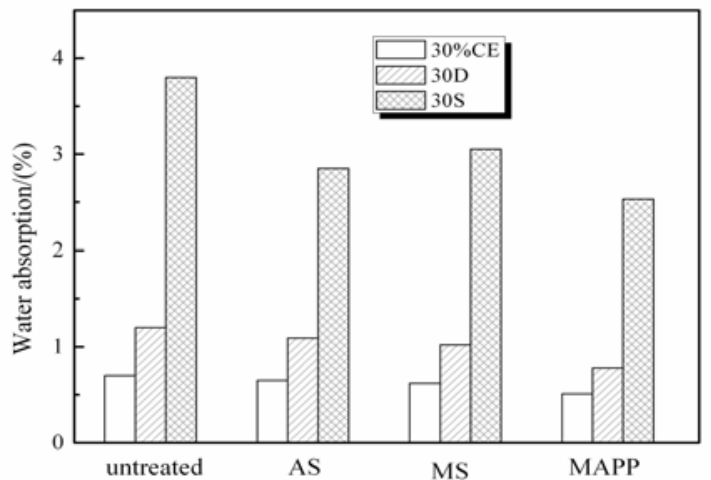

Fig. 2. Effect of coupling agents and fiber type on water sorption of composites

The decline in water sorption of composites with respect to fiber and coupling type is shown in Table.1. It was observed that MAPP treatment exhibited the best performance in terms of decreasing water absorption of the composites. All three coupling agents are capable of bonding to hydroxyl group of cellulose either by hydrogen or covalent bonds. Hydroxyl group reduction is accompanied by reduction in hydrophilicity. More hydrophobic nature yielded a decrease in water sorption of composites. Decrease in water absorption can be treated as an indicator of enhanced interfacial adhesion between fiber and matrix since tensile strength is in correlation with water sorption results. Thus MAPP treatment caused higher tensile strength compared to silane treatment. Comparison of water sorption results of three fiber types shows that WS is 3 and 5 times more capable of absorbing water compared to SD and CE, respectively. WS was also much more sensitive to coupling agents since enhancements in water sorption is much more pronounced compared to CE and WS. A possible cause of this observation could be greater porosity of WS fibers compared to CE and SD fibers. Fiber length is also greater in WS compared to SD. Void fraction experiments also showed that WS composites exhibited the highest void fraction. SD also has a higher void fraction than CE. Higher void fraction enables water molecules penetrate into the composite more easily. Coupling agents decreased void fraction which is a cause of restricted water penetration through matrix. Restriction of water penetration decreases water sorption of composites. This phenomenon can be treated as a dominating cause of the decrease in water sorption with coupling agent employment which is also interconnected with adhesion phenomenon.

Table.1. Decrease in water absorption of CE, SD and WS reinforced composites

\begin{tabular}{cccc}
\hline$\%$ decrease & AS & MS & MAPP \\
\hline CE & 8.5 & 7.8 & 20.5 \\
SD & 10.9 & 21.9 & 37.9 \\
WS & 25.1 & 22.1 & 49.8 \\
\hline
\end{tabular}


FTIR analysis of fibers and composites. Fig. 3 and Fig.4 shows FTIR spectra of CE, SD and WS at two different wave-number ranges. It can be seen there are additional peaks of WS and SD compared to $\mathrm{CE}$. It has to be emphasized that $\mathrm{CE}$ is a synthetic compound and only contains cellulose whereas SD and WS are natural compounds and may contain constituents other than cellulose. The peak around $1734 \mathrm{~cm}^{-1}$ is specific to SD and WS and assigned to $\mathrm{C}=\mathrm{O}$ stretching in hemicellulose. In addition, the peak specific to SD and WS at $1507 \mathrm{~cm}^{-1}$ is assigned to aromatic skeleton vibrations of lignin. The peaks observed for SD and WS at around $1460 \mathrm{~cm}^{-1}$ and $1420 \mathrm{~cm}^{-1}$ are assigned to $\mathrm{CH}$ deformation in lignin. These results prove that only constituent of CE is cellulose but WS and SD contains cellulose, lignin and hemicellulose in their chemical structure.

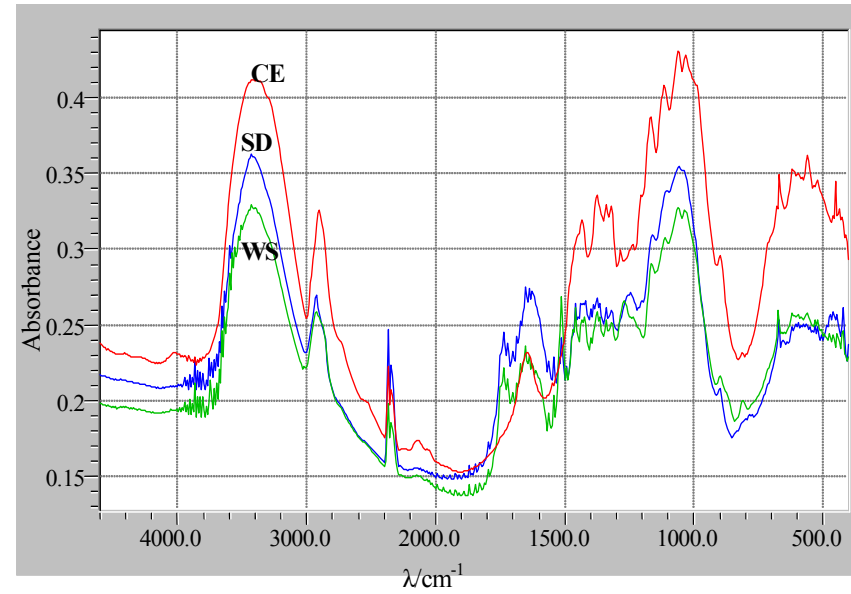

Fig. 3. FTIR spectra $\left(400-4400 \mathrm{~cm}^{-1}\right)$

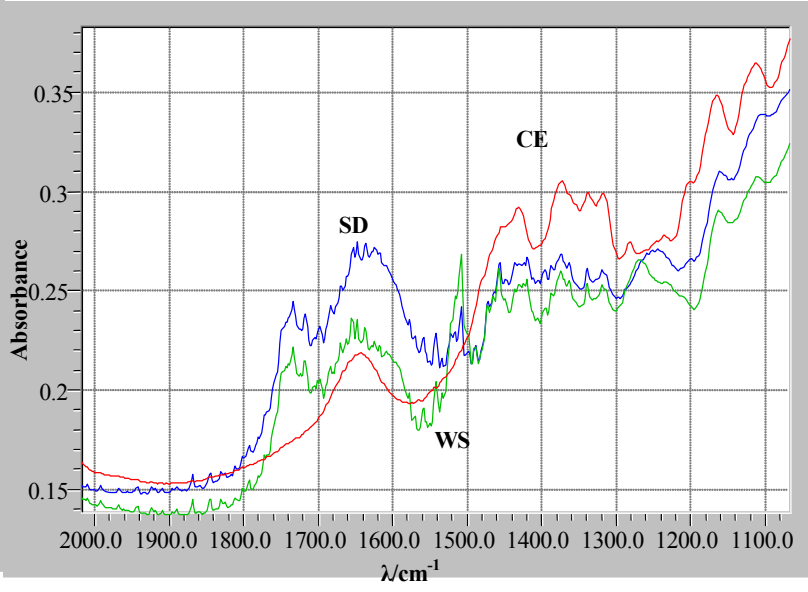

Fig. 4. FTIR spectra $\left(1100-2000 \mathrm{~cm}^{-1}\right)$

Fig. 5 shows FTIR spectra of untreated, $1 \mathrm{wt} \%$ AS and MS treated CE fibers. It can be seen that condensation reactions yields Si-O-Si linkage which have band at around $1135 \mathrm{~cm}^{-1}$. Si-O-Cellulose has peak at $1200 \mathrm{~cm}^{-1}$. In addition, $\mathrm{Si}-\mathrm{OCH}_{3}$ bonds have peaks around $1080-1100 \mathrm{~cm}^{-1}$. Si-OH groups which are products of hydrolization reactions have peak at $1015 \mathrm{~cm}^{-1}$. The band at $3400 \mathrm{~cm}^{-1}$ is caused by $\mathrm{OH}$ vibrations and can be treated as a measure of hydrophilicity. The band at $2990 \mathrm{~cm}^{-1}$ belongs to $\mathrm{C}-\mathrm{H}$ stretching of cellulose and do not change with silane treatment. Ratio of these two bands would be used to determine the relative degree of hydrophilcity to depict effect of coupling agents on hydrophilicity. As seen in Table.2, $\mathrm{I}_{3400} / \mathrm{I}_{2990}$ decreases with employment of silane coupling agents. The decrease is much more pronounced for AS treated CE. These results reveal that AS and MS are capable of decreasing hydrophilicity of CE.

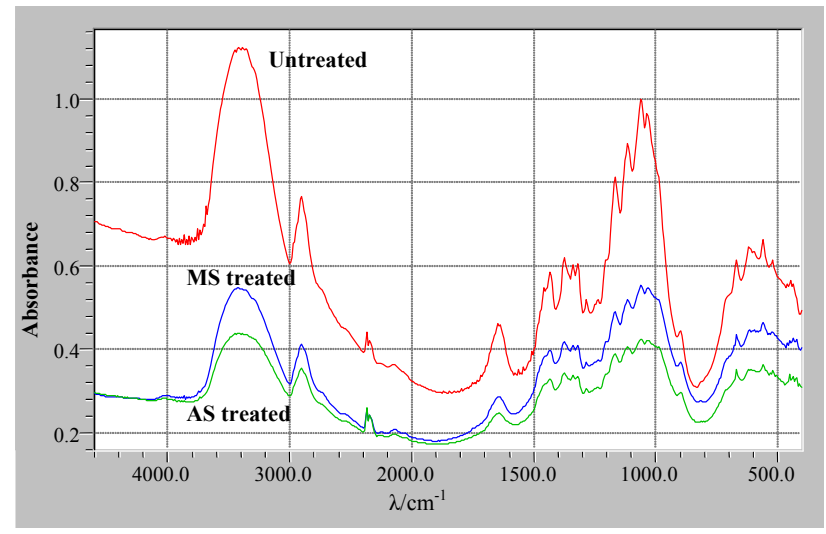

Table.2. Variation of $\mathrm{I}_{3400} / \mathrm{I}_{2990}$ with respect to silane coupling agents

\begin{tabular}{|c|c|}
\hline Treatment & $\mathrm{I}_{3400} / \mathrm{I}_{2990}$ \\
\hline Untreated CE & 2.74 \\
\hline $1 \mathrm{wt} \%$ AS treated CE & 2.17 \\
\hline $1 \mathrm{wt} \%$ MS treated CE & 2.45 \\
\hline
\end{tabular}

Fig. 5. FTIR spectra of CE, untreated and treated with $1 \mathrm{wt} \% \mathrm{AS}$ and MS

FTIR spectra of composites with AS and MS treatments did not exhibit any changes because of the 
reasons mentioned above but MAPP treatment in PP/CE composites at $30 \mathrm{wt} \%$ loadings, there were some new peaks associated with MAPP treatment due to stretching of carbonyl groups on MAPP ${ }^{[6]}$, as seen in Fig. 5.and Fig.6. The new bands observed are $1710 \mathrm{~cm}^{-1}$ characteristic of carbonyls from carboxylic dimer acid, 1785 and $1867 \mathrm{~cm}^{-1}$ characteristic of five-membered cyclic anhydride carbonyls. Activation of carbonyl groups leads ester linkages between cellulose and maleic anhdride group of MAPP, thus chemical coupling of cellulose to PP is achieved.

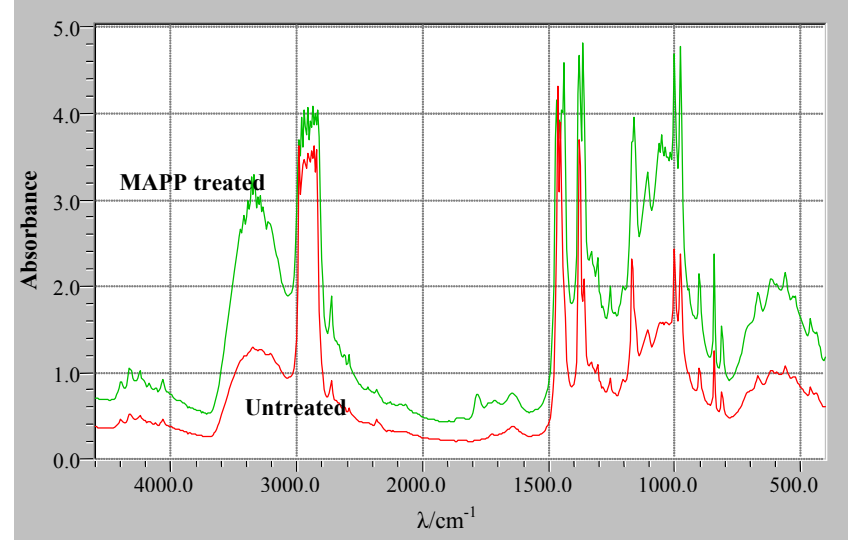

Fig.6. FTIR spectra of $30 \mathrm{wt} \% \mathrm{PP} / \mathrm{CE}$ composites $\left(400-4400 \mathrm{~cm}^{-1}\right)$

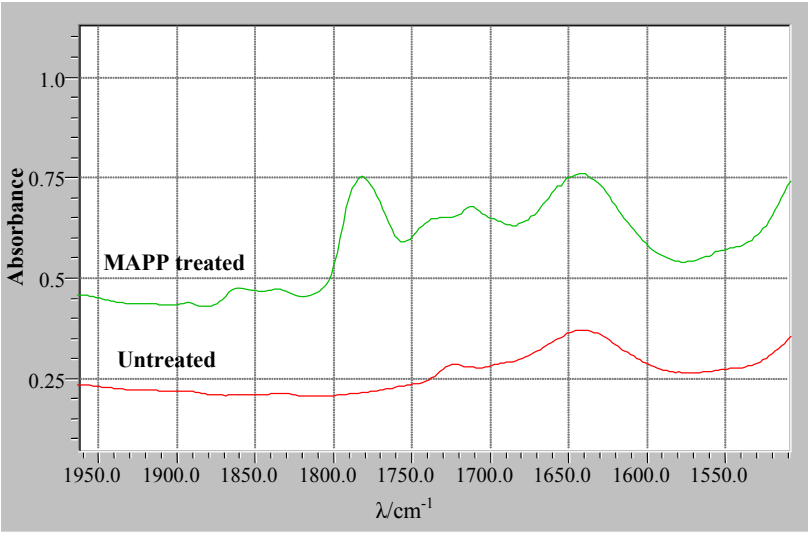

Fig.7. FTIR spectra of $30 \mathrm{wt} \% \mathrm{PP} / \mathrm{CE}$ composites $\left(1500-1950 \mathrm{~cm}^{-1}\right)$

\section{Conclusions}

Water absorption of composites increases in a linear fashion with increasing fiber loading. WS composites were capable of absorbing water 3-5 times more than SD and CE composites. MAPP performed superior performance in decreasing water sorption of composites. Up to $50 \%$ decrease in WS/PP composites was reported with employment of MAPP. AS and MS also exhibited decrease in water sorption. FTIR analysis put forward the decrease in hydrophilicity with silane treatment by comparing the band at $3400 \mathrm{~cm}^{-1}$, which belongs to $\mathrm{OH}$ groups on cellulose, and the band at 2990 which is a characteristic peak of cellulose. The ratio of these two bands decreased from 2.74 to 2.17 for AS treatment and to 2.45 for MS treatment. WS and SD contain hemicellulose and lignin other than cellulose whereas cellulose particulates were pure. New bands associated with carbonyl group on MAPP were determined when MAPP treated and untreated composites were compared.

\section{Acknowledgements}

The authors express their acknowledgements for the financial support provided by Taizhou Science and Technology Project (Grant No. 14GY02) and Maoming Science and Technology Project (Grant No. 915325), the Talent Recruitment Foundation from Guangdong University of Petrochemical Technology (Grant No. 650119), are highly appreciated.

\section{References}

[1] V. Fiore, T. Scalici, F. Nicoletti, G. Vitale: Composites Part B Vol. 85 (2016), p. 150

[2] G.A. Holt, P. Chow, J.D. Wanjura: Industrial Crops and Products Vol. 52(2014), p. 627

[3] D. Shanmugam, M. Thiruchitrambalam: Materials \& Design Vol. 50(2013), p. 533

[4] A. Ramzy, D. Beermann, L. Steuernagel, D. Meiners: Composites Part B Vol. 66(2014), p. 287

[5] B. Madsen, A. Thygsen, H. Lilholt: Composites Science and Technology Vol. 69(2009), p. 1057

[6] W. Qiu, F. Zhang, T. Endo, T. Hirotsu: Journal of Applied Polymer Science Vol. 91 (2004), p. 1703 